\title{
Lymphatic Transport of Stearic Acid and Its Effect on Cholesterol Transport in Rats
}

\author{
Ikuo IKEDA, * Youji IMASATo, Mioko NAKAYAMA, \\ Katsumi IMAIzUMI, and Michihiro SUGANO ${ }^{1}$ \\ Laboratory of Nutrition Chemistry and ${ }^{1}$ Laboratory of Food Science, \\ Kyushu University School of Agriculture, Fukuoka 812, Japan
}

(Received March 19, 1994)

\begin{abstract}
Summary Lymphatic transport of stearic acid, given as completely hydrogenated rapeseed oil (R10), 9 to 1 (R9) and 5 to 5 (R5) mixtures of R10, and soybean oil and completely hydrogenated tallow (T) was examined in the rat cannulated thoracic duct. R10, R9, R5, and T contained $91.4,81.5,46.5$, and $63.6 \%$ stearic acid, respectively. A large portion of the remaining fatty acids in $T$ was palmitic acid (31\%). These fats were emulsified with bile salt and albumin, and administered via a stomach tube. Lymphatic recovery of stearic acid at $24 \mathrm{~h}$ was lowest in $\mathrm{R}$ 10 and highest in R5, and intermediate in R9 and T. Recovery of oleic and linoleic acids in rats given R5 was almost complete and significantly higher than that of stearic acid. When $\mathrm{T}$ was given, the $24 \mathrm{~h}$ recovery of stearic acid was significantly lower than that of palmitic acid. A highly inverse correlation between the recovery and the content of stearic acid in administered fats was observed in R10, R9, and R5. Lymphatic recovery of cholesterol was almost parallel with that of stearic acid. Although the content of stearic acid in $T$ was lower than that in $R 9$, the recovery of stearic acid and cholesterol was almost similar. The results indicate that the rate of lymphatic recovery of stearic acid is affected by the quantity and quality of coexisting fatty acids.
\end{abstract}

Key Words stearic acid, lymph, absorption, fatty acids, cholesterol, rats, hydrogenated rape seed oil

Fats rich in saturated fatty acids are hypercholesterolemic and atherogenic in experimental animals and humans (1). However, cocoa butter, a fat rich in saturated fatty acids, has been reported to be less cholesteremic in experimental animals (2) in contrast to the well known multiple regression $(3,4)$. At least a part of the effect of cocoa butter has been ascribed to the low absorbability of stearic acid and its inhibitory effect on cholesterol absorption (5). Although many studies reported that intestinal absorption of stearic acid was lower than that of the

* To whom all correspondence should be addressed. 
unsaturated fatty acids with the same chain length, wide variations of the absorption rate were observed depending on the experiments (5-9). There is a possibility that the variation in stearic acid absorption is due to the quantity and quality of coexisting fatty acids as well as the triglyceride structure of dietary fat. However, the characteristics of stearic acid absorption are not understood in detail. Utilization of less absorbable and non-cholesteremic stearic acid make it possible to develop low calorie substitutes.

In this study, the effect of a graduated addition of soybean oil on the lymphatic recovery of completely hydrogenated rapeseed oil containing more than $90 \%$ stearic acid and cholesterol recovery was examined in rats with a cannulated thoracic duct. The recovery of completely hydrogenated tallow, containing $64 \%$ stearic acid and $31 \%$ palmitic acid, was also measured.

\section{EXPERIMENTAL}

Materials. $\left[4-{ }^{14} \mathrm{C}\right]$ Cholesterol $(60 \mathrm{mCi} / \mathrm{mmol})$ was purchased from NEN Research Products (Boston, MA, USA) and purified by thin-layer chromatography on benzene/ethylacetate $(7: 3, \mathrm{v} / \mathrm{v})$ prior to use. Fatty acid-free bovine serum albumin fraction $\mathrm{V}$ was obtained from Miles Inc. (Kankakee, IL, USA). Sodium taurocholate (purity $>98 \%$ ) and cholesterol were from Nacalai Tesque (Kyoto). Completely hydrogenated rapeseed oil, soy bean oil, and completely hydrogenated Tallow were kindly provided by Nippon Oil \& Fats Co. (Tokyo). Fatty acid composition of these fats and the 9 to 1 and 5 to 5 mixtures of completely hydrogenated rapeseed oil and soy bean oil analyzed by gas-liquid chromatography (GLC) is shown in Table 1.

Animals and treatments. Male Sprague-Dawley rats (Seiwa Experimental Animals, Fukuoka) weighing 300-320 g were fed a nonpurified diet (type NMF, Oriental Yeast, Tokyo) and drinking water ad.libitum. A cannula was inserted into the left thoracic lymphatic channel of each rat and an indwelling catheter was placed in the stomach (10). Normal osmotic glucose (139 mM)- $\mathrm{NaCl}(85 \mathrm{~mm})$ solution was infused continuously $(3 \mathrm{ml} / \mathrm{h})$ until the end of lymph collection. After surgery, each rat was placed in a restraining cage in a warm recovery room and allowed free access to drinking water containing $139 \mathrm{mM}$ glucose and $85 \mathrm{mM} \mathrm{NaCl}$.

Table 1. Fatty acid composition of the administered fats.

\begin{tabular}{lrrrrr}
\hline Fat & $16: 0$ & $18: 0$ & $18: 1$ & $18: 2$ & $18: 3$ \\
\hline R10 & 4.4 & 91.4 & 1.0 & - & - \\
R9 & 4.7 & 81.5 & 3.7 & 5.6 & 1.1 \\
R5 & 6.9 & 46.5 & 12.8 & 26.5 & 4.3 \\
T & 31.0 & 63.6 & 0.6 & - & - \\
\hline
\end{tabular}

$\mathrm{R} 10$, hydrogenated rapeseed oil; R9, a mixture of R10 and soybean oil (9:1); R5, a mixture of R10 and soybean oil $(5: 5)$; T, hydrogenated tallow. 
During the next morning, after collection of lymph for $2 \mathrm{~h}$ (blank lymph), each animal was administered $3 \mathrm{ml}$ of a test emulsion via a stomach tube, and the lymph was collected for $24 \mathrm{~h}$ beginning at $10 \mathrm{a} . \mathrm{m}$. The test emulsion contained $200 \mathrm{mg}$ of sodium taurocholate, $50 \mathrm{mg}$ of fatty acid-free albumin, $1 \mu \mathrm{Ci}$ of $\left[4-{ }^{14} \mathrm{C}\right] \mathrm{cholesterol}$ and $200 \mathrm{mg}$ of fat. The emulsion was prepared by sonication after heating the fats in a water bath, and was immediately administered to rats. Lymph was collected in an EDTA-containing tube at 3-h intervals during the first $9 \mathrm{~h}$ and at a single collection 9-24 $\mathrm{h}$ after administration of a test emulsion.

Measurement of cholesterol and fatty acid absorption. A 1-ml aliquot of lymph was transferred into a scintillation vial and $10 \mathrm{ml}$ of Aquasol II (NEN Research Products) was added. The radioactivity was counted using an Aloka liquid scintillation counter (LSC-1000, Tokyo). Another aliquot of lymph was extracted in 20 volumes of chloroform : methanol $(2: 1, \mathrm{v} / \mathrm{v})$, and pentadecanoic acid (Aldrich, Milwaukee, WI) was added as an internal standard. Quantification of fatty acids was performed by GLC as described previously (11). Fatty acid absorption was calculated by subtracting fatty acid amounts in blank lymph from those of lymph collected after administration of a test emulsion.

Statistical analysis. Statistical difference among each group was determined by Duncan's new multiple-range test (12) and Student's $t$-test (13).

\section{RESULTS}

Lymph flow rates were linear for $24 \mathrm{~h}$ in all groups and tended to be lower in

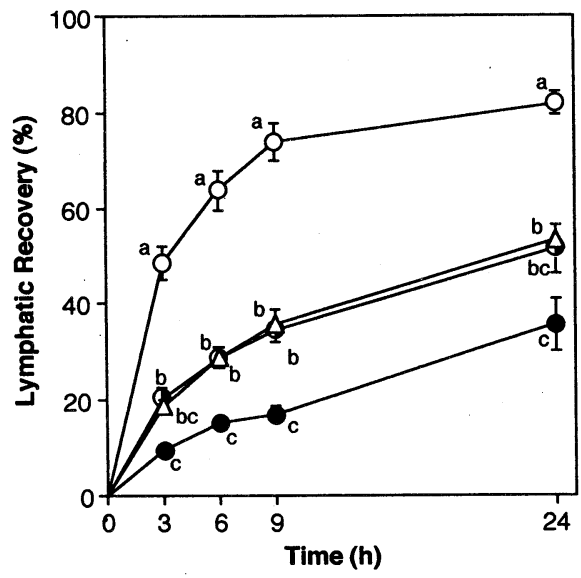

Fig. 1. Recovery of stearic acid in the thoracic duct lymph of rats after intragastric administration of fat emulsions containing sodium taurocholate, albumin, and labeled cholesterol. $\bullet, \mathbf{R} 10$ group; $\mathbf{O}, \mathbf{R} 9$ group; $\bigcirc, \mathbf{R} 5$ group; $\triangle, \mathbf{T}$ group. $\mathrm{R} 10$, hydrogenated rapeseed oil; $\mathrm{R} 9$ and $\mathrm{R} 5$, a mixture of $\mathrm{R} 10$ and soybean oil = $9: 1$ and 5:5, respectively; T, hydrogenated tallow. Data are $\mathrm{M} \pm \mathrm{SE}$ of 6 rats. Values with different letters are significantly different at $p<0.05$. 
rats given $R 9(110 \pm 10 \mathrm{ml} / 24 \mathrm{~h})$ and $\mathrm{R} 10(114 \pm 9 \mathrm{ml} / 24 \mathrm{~h})$ than in those given $\mathrm{R} 5$ $(140 \pm 7 \mathrm{ml} / 24 \mathrm{~h})$ and $\mathrm{T}(159 \pm 18 \mathrm{ml} / 24 \mathrm{~h})$.

Lymphatic recovery of stearic acid for $24 \mathrm{~h}$ was considerably low $(35.6 \pm 5.6 \%$ for $24 \mathrm{~h}$ ) when R10 was given (Fig. 1). The addition of soybean oil significantly improved the recovery up to $51.4 \pm 5.2$ and $81.8 \pm 2.3 \%$ in rats given $R 9$ and $R 5$, respectively. The $24 \mathrm{~h}$ recovery of stearic acid given as $\mathrm{T}(53.4 \pm 3.1 \%)$ was similar

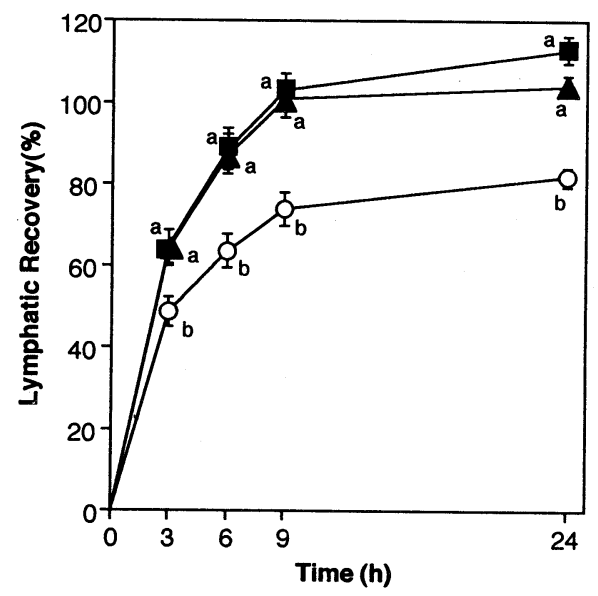

Fig. 2. Recovery of stearic, oleic, and linoleic acids in the thoracic duct lymph of rats after intragastric administration of the fat emulsion containing R5. See also legend of Fig. 1. $\bigcirc$, stearic acid; $\Delta$, oleic acid; $\mathbf{\square}$, linoleic acid. Data are $\mathbf{M} \pm$ $\mathrm{SE}$ of 6 rats. Values with different letters are significantly different at $p<0.05$.

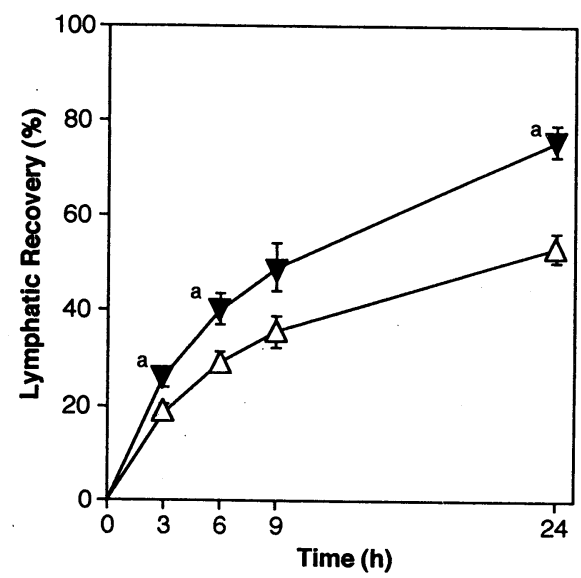

Fig. 3. Recovery of stearic and palmitic acids in the thoracic duct lymph of rats after intragastric administration of the fat emulsion containing $T$. See also legend of Fig. 1. $\triangle$, stearic acid; $\boldsymbol{\nabla}$, palmitic acid. Data are $\mathbf{M} \pm \mathrm{SE}$ of 6 rats. a, significantly higher than the corresponding stearic acid recovery $(p<0.05)$. 
to that in R9. A high inverse linear correlation between stearic acid content of the administered fats and stearic acid absorption was observed in R10, R9, and R5 ( $r=$ $-0.99, p<0.01)$.

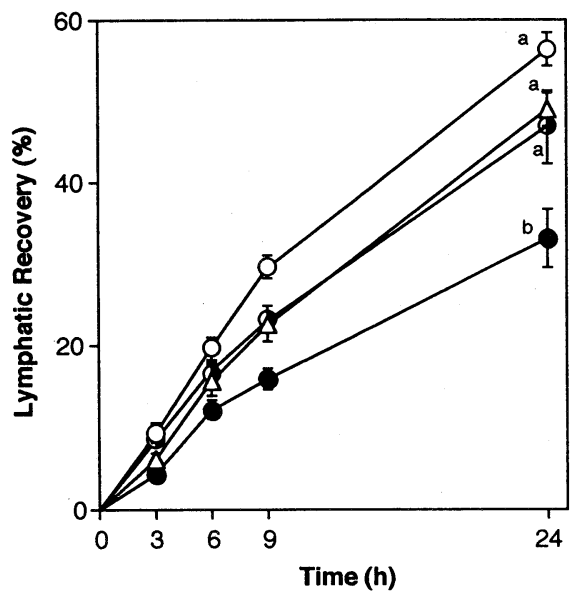

Fig. 4. Recovery of cholesterol in the thoracic duct lymph of rats after intragastric administration of the fat emulsion containing sodium taurocholate, albumin, and labeled cholesterol. - $\mathrm{Q} 10$ group; $\mathbf{O}, \mathrm{R} 9$ group; $\bigcirc, \mathrm{R} 5$ group; $\triangle$, T group. See also legend of Fig. 1. Data are $\mathrm{M} \pm \mathrm{SE}$ of 6 rats. Values with different letters are significantly different at $p<0.05$.

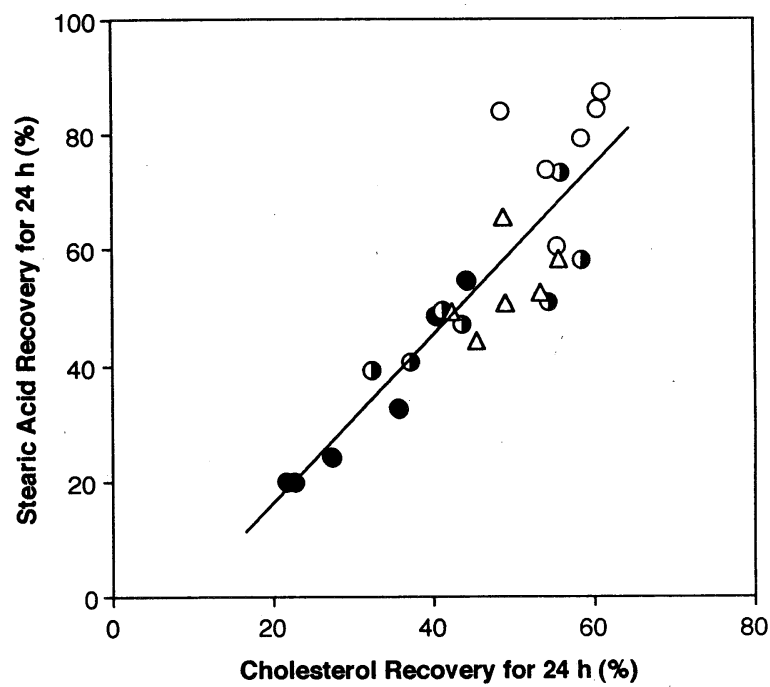

Fig. 5. Correlation of lymphatic recovery of stearic acid with cholesterol. $\bullet, R 10$ group; $\mathbf{O}, \mathbf{R} 9$ group; $\bigcirc, \mathbf{R} 5$ group; $\triangle, \mathbf{T}$ group. See also legend of Fig. 1. A highly positive linear correlation $(r=0.87)$ was observed $(p<0.01)$. 
As shown in Fig. 2, the 24-h recovery of oleic and linoleic acids in rats given R5 was almost quantitative and was significantly higher than that of stearic acid. The recovery of palmitic acid given as $\mathrm{T}$ was significantly higher than that of stearic acid from the same fat (Fig. 3). However, the 24-h recovery of palmitic acid was considerably lower than that of oleic and linoleic acids in the R5 fat (compare Fig. 2 and Fig. 3).

Lymphatic transport of cholesterol given with the fats exhibited almost the same pattern as that of stearic acid (Fig. 4). The recovery of cholesterol was highest in the R5 fat and lowest in the R10 fat. Cholesterol absorption in rats given R9 and T fats was found to be intermediate. A highly positive correlation was observed between the lymphatic recoveries of stearic acid and cholesterol (Fig. 5).

\section{DISCUSSION}

Our study showed that lymphatic recovery of stearic acid is dependent on the quantity and quality of coexisting fatty acids. Although the fats administered were well emulsified with bile salt and albumin in this experiment, the recovery for $24 \mathrm{~h}$ of stearic acid given as R10 was extremely low. The recovery of stearic acid increased as the proportion of soybean oil in R10 increased. A linear inverse correlation between the stearic acid content in fats and stearic acid recovery in lymph was observed. It is thought that fats having a higher melting point may interfere with the formation of fat emulsion in the stomach, accessibility of lipase to the fat emulsion and/or micellar solubility of the hydrolysis products (14). Therefore, the increased recovery of stearic acid by the increased addition of soybean oil is at least partly dependent on lowering of the melting point of the fats administered. Although stearic acid content in R9 (82\%) was higher than that in $\mathrm{T}(64 \%)$, the absorption of stearic acid was comparable between these two fats. This suggests that the coexistence of palmitic acid may not improve the absorption of stearic acid, while the addition even in a small amount of unsaturated fatty acids such as oleic and linoleic acid is more effective in increasing stearic acid absorption.

Alternatively, there is a possibility that pancreatic lipase does not specifically hydrolyze glycerides containing stearic acid at the 1 and 3 positions. However, since the addition of soybean oil to R10 improved the lymphatic recovery of stearic acid, it is more plausible that melting point, rather than fatty acid specificity of lipase, determines the absorbability of stearic acid.

Uptake of fatty acids by intestinal brush border membranes and resynthesis to triglyceride in intestinal cells are also important factors influencing fatty acid absorption. Yang et al. suggested that incorporation of monoglycerides into mucosal cells might be altered by the type of fatty acids bound to glycerol (15). Bergstedt et al. also demonstrated that stearic acid is taken up and transported by the small intestine less efficiently than oleic acid $(8,16)$.

Mattson reported that there is an inverse linear relationship between the tristearin content of dietary fat and the coefficient of the absorbability assessed by 
fecal excretion of stearic acid (17) and this is consistent with our results. The percentage absorption of stearic acid in our experiment is considerably higher than that in his results, when comparison was made with the same content of stearic acid in dietary fat. The difference can be explained by a difference in the experimental conditions. The diet containing stearic acid as triglyceride was fed to rats in his experiment, whereas the fats were emulsified with sonication and immdiately administered in this experiment. Therefore, emulsification of fats in stomach was bypassed in the present study.

In rats given $\mathrm{R} 5$, lymphatic recovery of stearic acid was clearly lower than that of the coexisting oleic and linoleic acids, and the recovery of these unsaturated fatty acids during the $24-\mathrm{h}$ period was almost quantitative. The results suggests that the existence of stearic acid in the intestinal lumen does not interfere with the absorption of the coexisting unsaturated fatty acids.

It is known that dietary fat accelerates cholesterol absorption (11), since hydrolysis products of triglyceride by lipase in the intestinal lumen, monoglyceride and free fatty acid, solubilize more cholesterol in mixed micellar solution. Therefore, a lower degree of hydrolysis of stearic acid-containing triglyceride by lipase together with the limited solubility of hydrolysis products in bile salt micelles may be the reason for the low absorption of cholesterol in fats containing stearic acid.

Positional specificity of fatty acids in triglyceride may also influence digestion and absorption of fatty acids (18). In this context, Mattson reported that the absorption coefficient of stearic acid in the randomized fat consisting of tristearin and safflower oil was different from that in the simple mixture (17). It was also reported that the structural difference of triglycerides containing stearic and oleic acids is an important factor influencing the absorption of stearic acid, although oleic acid absorption was almost complete irrespective of the triglyceride source (19). In contrast, the absorption of palmitic acid in native and randomized palm oil was similar (20). Thus, further detailed study is necessary in this regard.

In conclusion, stearic acid may have a hypocholesterolemic effect through less absorbability and inhibitory effect on cholesterol absorption. However, these effects of stearic acid are largely influenced by feeding conditions as well as the quantity and quality of coexisting fatty acids. A relatively high intake of stearic acid may be necessary for effective utilization.

\section{REFERENCES}

1) Shore, V. G., Butterfield, G., and Krauss, R. M. (1983): Effects of varying the dietary ration of polyunsaturated to saturated fats on plasma lipid and liporoteins, in Dietary Fats and Health, ed. by Perkins, E. G., and Visek, W. J., AOCS, Champaign, pp. 667678.

2) Kritchevsky, D., Tepper, S. A., Bises, G., and Klurfeld, D. M. (1982): Experimental atherosclerosis in rabbits fed cholesterol-free diets. 10. Cocoa butter and palm oil. Atherosclerosis, 41, 279-284. 
3) Keys, A., and Parlin, R. W. (1966): Serum-cholesterol response to changes in dietary lipids. Am. J. Clin. Nutr., 19, 175-181.

4) Hegsted, D. M., McGandy, R. B., Myers, M. L., and Stare, F. J. (1965): Quantitative effects of dietary fat on serum cholesterol in man. Am. J. Clin. Nutr., 17, 281-295.

5) Chen, I. S., Subramaniam, S., Vahouny, G. V., Cassidy, M. M., Ikeda, I., and Kritchevsky, D. (1989): A comparison of the digestion and absorption of cocoa butter and palm kernel oil and their effects on cholesterol absorption in rats. J. Nutr., 119, 1569-1573.

6) Bonanome, A., and Grundy, S. M. (1989): Intestinal absorption of stearic acid after consumption of high fat meals in humans. J. Nutr., 119, 1556-1560.

7) Denke, M., and Grundy, S. M. (1991): Effect of fats high in stearic acid on lipid and lipoprotein concentrations in men. Am. J. Clin. Nutr., 54, 1036-1040.

8) Bergstedt, S. E., Hayashi, H., Kritchevsky, D., and Tso, P. (1990): A comparison of absorption of glycerol tristearate and glycerol trioleate by rat small intestine. Am. J. Physiol., 259, G386-G393.

9) Jones, P. J. H., Pencharz, P. B., and Clandinin, M. T. (1985): Absorption of ${ }^{13}$ Clabeled stearic, oleic, and linoleic acids in humans; Application to breath test. J. Lab. Clin. Med., 105, 647-652.

10) Ikeda, I., Imasato, Y., Nagao, H., Sasaki, E., Sugano, M., Imaizumi, K., and Yazawa, K. (1993): Lymphatic transport of eicosapentaenoic and docosahexaenoic acids as triglyceride, ethyl ester and free acid, and their effect on cholesterol transport in rats. Life Sci., 52, 1371-1379.

11) Ikeda, I., Tomari, Y., and Sugano, M. (1989): Interrelated effects of dietary fiber and fat on lymphatic cholesterol and triglyceride absorption in rats. J. Nutr., 119, 13831387.

12) Duncan, D. B. (1955): Multiple range and multiple $F$ tests. Biometrics, 11, 1-42.

13) Fisher, R. A. (1970): Statistical Methods for Research Workers, Vol. 14, Oliver and Boyd, Edinburgh, pp. 140-142.

14) Nelson, G. J., and Ackman, R. G. (1988): Absorption and transport of fat in mammals with emphasis on $n-3$ polyunsaturated fatty acids. Lipids, 23, 1005-1014.

15) Yang, L. Y., Kuksis, A., and Myher, J. J. (1990): Intestinal absorption of menhaden and rapeseed oils and their fatty acid methyl and ethyl esters in the rat. Biochem. Cell Biol., 68, 480-491.

16) Bergstedt, S. E., Bergsted, J. L., Fujimoto, K., Mansbach, C., Kritchevsky, D., and Tso, P. (1991): Effect of glycerol tripalmitate and glycerol trioleate on intestinal absorption of glycerol tristearate. Am. J. Physiol., 261, G239-G247.

17) Mattson, F. H. (1959): The absorbability of stearic acid when fed as a simple or mixed triglyceride. J. Nutr., 69, 338-342.

18) Small, D. M. (1991): The effects of glyceride structure on absorption and metabolism. Annu. Rev. Nutr., 11, 413-434.

19) Mattson, F. H., Nolen, G. A., and Webb, M. R. (1979): The absorbability by rats of various triglycerides of stearic and oleic acid and the effect of dietary calcium and magnesium. J. Nutr., 109, 1682-1687.

20) Sugano, M., Ikeda, I., Imasato, Y., Nakayama, M., and Yoshida, K. (1990): Is triglyceride structure of palm oil responsible for its characteristic effect on lipid metabolism? Life Sci. Adv. Pharm., 9, 21-25. 